\title{
A STRONG FORM OF THE PHRAGMEN-BROUWER THEOREM
}

\author{
R. F. DICKMAN, JR.
}

\begin{abstract}
In this paper we prove the following form of the Phragmen-Brouwer Theorem: a locally connected, connected normal $T_{1}$-space $X$ is unicoherent if and only if for every pair of disjoint nonseparating continua $C$ and $D$ in $X, C \cup D$ does not separate $X$. Among the several corollaries is the proposition: $X$ is multicoherent if and only if $X$ is the union of a circular chain of continua $\left\{A_{0}, A_{1}, A_{2}, A_{3}\right\}$ where no three of the $A_{i}$ 's have a point in common.
\end{abstract}

1. Introduction. Throughout this paper $X$ will denote a normal, locally connected, connected $T_{1}$-space. By a continuum we mean a closed and connected (not necessarily compact) subset of $X$, a region is an open connected subset of $X$. For $A \subset X$, $b_{0}(A)$ denotes the number of components of $A$ less one (or $\infty$ if this number is infinite). The degree of multicoherence, $r(X)$, of $X$ is defined by

$$
r(X)=\sup \left\{b_{0}(H \cap K): X=H \cup K \text { and } H \text { and } K \text { are subcontinua of } X\right\} .
$$

If $r(X)=0, X$ is said to be unicoherent and we say that $X$ is multicoherent otherwise. If $0<r(X)<\infty$, we say that $X$ is finitely multicoherent and if $0<r(X) \leqslant \infty$ but $b_{0}(H \cap K)<\infty$ for any representation $X=H \cup K$, where $H$ and $K$ are continua, we say that $X$ is weakly-finite multicoherent.

A connected subset $C$ of $X$ is said to be simple if $X \backslash C$ is also connected.

Unicoherence is characterized in the Phragmen-Brouwer Theorem (PB Theorem) as follows (see [10, Theorem (3.2)]):

A connected, locally connected, normal space $X$ is unicoherent if and only if whenever $A$ and $B$ are disjoint closed sets and neither $A$ nor $B$ separates $X$, then $A \cup B$ does not separate $X$.

J. H. V. Hunt extended the PB Theorem to arbitrary locally connected topological spaces in [7], and he and E. D. Tymchatyn were able to replace "closed" by "arbitrary set" in [8]. The PB Theorem has been generalized by A. H. Stone in [11, Theorem 5 and 13, Theorem 6] to multicoherent spaces, i.e. to spaces $X$, where $1 \leqslant r(X)$.

The principal result of this paper is the following:

A connected, locally connected normal $T_{1}$-space $X$ is unicoherent if and only if whenever $C$ and $D$ are disjoint simple continua in $X, X \backslash(C \cup D)$ is connected.

Received by the editors October 20, 1982 and, in revised form, May 31, 1983. 1980 Mathematics Subject Classification. Primary 54F55; Secondary 54F25.

Key words and phrases. Unicoherence, circular chain of continua.

(C)1984 American Mathematical Society $0002-9939 / 84 \$ 1.00+\$ .25$ per page 
This proposition was known to hold for compact spaces or weakly-finitely multicoherent spaces [5]; in [4], a proof for this proposition, when $X$ is assumed to be perfectly normal, was given.

We call the proposition above "a strong form" of the PB Theorem because in the sufficiency (i.e. the if part), we have weakened the hypothesis to requiring that $A$ and $B$ be continua, while essentially retaining the general setting (connected, locally connected and normal) of the PB Theorem. The reader is referred to [7] for additional comments on the nomenclature of the "Phragmen-Brouwer Theorem".

\section{Preliminary results.}

Definitions and Notation. Let $A$ and $B$ be nonempty, disjoint closed subsets of $X$. A division of $X$ with respect to $A$ and $B$ is a representation $X=R \cup F \cup S$, where $R$ and $S$ are disjoint regions, $\operatorname{Fr} R=\operatorname{Fr} S, \operatorname{Fr} R \subseteq F, \operatorname{Fr} R \cap(A \cup B)=\varnothing$ and $A \cap R \neq \varnothing \neq B \cap S$ (cf. [14, p. 49]).

Lemma (2.1). Let $A$ and $B$ be disjoint closed subsets of $X$ and let $a \in A$ and $b \in B$. Then there exists a division $X=R \cup F \cup S$ such that $a \in R, b \in S$, and $R$ and $S$ are simple regions in $X$. Furthermore, if $A$ (respectively, $B$ ) is a continuum, then $A \cap F=$ $\varnothing$ (respectively, $B \cap F=\varnothing)$.

Proof. Let $U$ be an open subset of $X$ such that $A \subseteq U \subseteq \bar{U} \subseteq X \backslash B$, let $P$ be the component of $U$ containing $a$, and let $S$ be the component of $X \backslash \bar{P}$ containing $b$ and let $F=X \backslash(R \cup S)$. Then since $X$ is locally connected, $\operatorname{Fr} R=\operatorname{Fr} S \subseteq \operatorname{Fr} U \subseteq$ $X \backslash(A \cup B)$. Also, since $X$ is connected, every component of $X \backslash(\bar{R} \cup \bar{S})$ has a limit point in $\operatorname{Fr} R$. This implies that $X \backslash S=R \cup F$ and $X \backslash R=S \cup F$ are connected sets, as required. Finally, if $A$ is a continuum, $A \subseteq R$ since $A \cap R \neq \varnothing$ and $A \cap \operatorname{Fr} R=\varnothing$; hence $A \cap F=\varnothing$. This completes the proof of Lemma (2.1).

\section{Main result.}

Theorem (3.1) (Strong form of the Phragmen-Brouwer Theorem). A connected, locally connected normal $T_{1}$-space is unicoherent if (and only if) whenever $C$ and $D$ are disjoint simple continua in $X$, then $X /(C \cup D)$ is connected.

Proof. Suppose that if neither of two disjoint continua separate $X$, then their union does not separate $X$ and suppose that $X$ is not unicoherent. Then there exist continua $H$ and $K$ such that $X=H \cup K$ and $H \cup K=A \cup B$ where $A$ and $B$ are nonempty disjoint closed sets. Let $a \in A$ and $b \in B$. By Lemma (2.1) there exists a division $X=R \cup F \cup S$ where $a \in R, b \in S$ and $\operatorname{Fr} R \cap(A \cup B)=\varnothing$.

Let $\mathcal{C}=\left\{\bar{S} \cap \bar{R}^{\prime}: X=R^{\prime} \cup F^{\prime} \cup S^{\prime}\right.$ is a division of $X, \bar{R} \subseteq R^{\prime}, b \in S^{\prime}$ and $\left.\operatorname{Fr} R^{\prime} \cap(A \cup B)=\varnothing\right\}$. We assert that $\cap \mathcal{C}=F r S$. It is clear that $\operatorname{Fr} S \subseteq \cap \mathcal{~}$. Suppose $x \notin \operatorname{Fr} S$; we will argue that $x \notin \cap \mathcal{C}$. Since $x \notin \operatorname{Fr} S, x \in(X \backslash \bar{S})$ or $x \in S$. If $x \in X \backslash \bar{S}$, then $X \backslash \bar{S}$ is an open set containing $x$ that misses $\cap \mathcal{C}$. Hence, in the first case $x \notin \cap \mathcal{C}$. On the other hand suppose $x \in S$. Then since $X$ is a locally connected normal $T_{1}$-space, there is a continuum $B_{0}$ containing $\{x, b\}$ and such that $B_{0} \subseteq S$. Then by Lemma (2.1), there is a division $X=R^{\prime \prime} \cup F^{\prime \prime} \cup S^{\prime \prime}$ such that $\bar{R} \subseteq R^{\prime \prime}, B_{0} \subseteq S^{\prime \prime}$ and Fr $R^{\prime \prime} \cap\left(A \cup B \cup B_{0}\right)=\varnothing$. Then $x \in S^{\prime \prime}$ and $S^{\prime \prime} \cap(\bar{S}$ $\left.\cap \bar{R}^{\prime \prime}\right)=\varnothing$. Hence $S^{\prime \prime}$ misses $\cap \bigodot$ and so $\operatorname{Fr} S=\cap \bigodot$. 
Let $\beta X$ denote the Stone-Ĉech compactification of $X$ and $\operatorname{cl}_{\beta} S$ denote the closure in $\beta X$ of a subset $S$ of $\beta X$. Let $T=\operatorname{cl}_{\beta}(A \cup B)$ and let $D=\operatorname{cl}_{\beta} \operatorname{Fr} S$. Since Fr $S \cap(A \cup B)=\varnothing, \operatorname{Fr} S$ and $A \cup B$ are completely separated subsets of $X$ and $D \cap T=\varnothing[9$, p. 86]. Now $D$ contains Fr $S$ and this implies that $D$ meets each of the disjoint open sets $U=\left(\operatorname{cl}_{\beta} H\right) \backslash(T)$ and $V=\left(\operatorname{cl}_{\beta} K\right) \backslash T$. Since $\beta X \backslash(U \cup V)$ is compact, there exist divisions $X=R_{i} \cup F_{i} \cup S_{i}, i=1, \ldots, n$, such that $\bar{R} \subseteq R_{i}$, $b \in S_{i}$ and $\operatorname{Fr} R_{i} \cap(A \cup B)=\varnothing$ and $\beta X \backslash(U \cup V) \subseteq \cup_{i=1}^{n}\left\{\beta X \backslash \operatorname{cl}_{\beta}\left(S \cap R_{i}\right)\right\}$.

Since $b \in \cap_{i=1}^{n} S_{i}, \cup_{i=1}^{n} \bar{S}_{i}$ is a continuum and by Lemma (2.1) there exists a division $X=R_{0} \cup F_{0} \cup S_{0}$ such that $\bar{R} \subseteq R_{0}, \cup_{i=1}^{n} \bar{S}_{i} \subseteq S_{0}$ and $\operatorname{Fr} R_{0} \cap(A \cup B)$ $=\varnothing$. It then follows that $\operatorname{cl}_{\beta}\left(S \cap R_{0}\right) \subseteq \cap_{i=1}^{n} \operatorname{cl}_{\beta}\left(S \cap R_{i}\right)$ and so $\beta X \backslash(U \cup V) \subseteq$ $\beta X \backslash \mathrm{cl}_{\beta}\left(S \cap R_{0}\right)$. By our hypothesis $S \cap R_{0}=[X \backslash(R \cup F)] \cap\left[X \backslash\left(S_{0} \cup F_{0}\right)\right]=$ $X \backslash\left[(R \cup F) \cup\left(S_{0} \cup F_{0}\right)\right]$ is a connected set as in $\operatorname{cl}_{\beta}\left(S \cap R_{0}\right)$. Of course this means the connected set $\operatorname{cl}_{\beta}\left(S \cap R_{0}\right)$ meets each of $U$ and $V$ and thus $\operatorname{cl}_{\beta}\left(S \cap R_{0}\right)$ cannot be a subset of $U \cup V$. This contradiction implies that $X$ must be unicoherent and this completes the proof.

REMARK. Note that in the proof of Theorem (3.1), the closedness of the points of $X$ was employed twice: first in the continuumwise connectivity of the region $S$ so as to obtain the subcontinuum $B_{0}$ above, and secondly in the existence of $\beta X$. In regard to the latter use, we could have employed the well-known construction of the Tychonoff space $Y$ and the continuous surjection $\sigma: X \rightarrow Y$, where the correspondence $f \rightarrow f \circ \sigma$ is an isomorphism of $C(Y)$ onto $C(X)$, and avoided the necessity of assuming points are closed. However, the author has not been able to obtain a comparable substitution in the first use of $T_{1}$-ness above.

The following proposition follows from the above theorem and [6, Theorem 1 and 5, Theorem 2], albeit via a rather circuitous route. A proof is provided herein for completeness.

COROLLARY (3.2). The following are equivalent:

(i) $X$ is multicoherent.

(ii) There exist simple continua $A_{0}, A_{1}, A_{2}$ and $A_{3}$ such that

(a) $X=A_{0} \cup A_{1} \cup A_{2} \cup A_{3}$,

(b) no three of the A's have a point in common,

(c) $A_{i} \cap A_{j} \neq \varnothing$ if and only if $|(i-j) \bmod 4| \leqslant 1$.

(iii) There exist nonempty disjoint simple continua $B_{1}$ and $B_{2}$ such that $B_{1} \cup B_{2}$ separates $X$.

Proof. We shall show (i) implies (ii). Suppose $X$ is multicoherent. Then, by Theorem (3.1), there exist simple continua $C$ and $D$ such that $X \backslash(C \cup D)=P \cup Q$ where $P$ and $Q$ are nonempty disjoint open sets. By Lemma (2.1) there exist divisions $X=R_{i} \cup F_{i} \cup S_{i}, 0 \leqslant i \leqslant 2$, such that $C \subseteq R_{0} \cap R_{1} \cap R_{2}, D \subseteq S_{0} \cap S_{1}$ $\cap S_{2},\left(R_{1} \cup F_{1}\right) \subseteq R_{0} \subseteq\left(R_{0} \cup F_{0}\right) \subseteq R_{2}$.

Since $C$ and $D$ are simple sets and since $R_{1} \cup F_{1}$ and $S_{1} \cup F_{2}$ are simple sets, every component of

$$
P \cap\left[X \backslash\left(R_{1} \cup F_{1} \cup S_{2} \cup F_{2}\right)\right] \quad \text { and } \quad Q \cap\left[X \backslash\left(R_{1} \cup F_{1} \cup S_{2} \cup F_{2}\right)\right]
$$


has a limit point in each of $\bar{R}_{1}$ and $\bar{S}_{2}$. Similarly, we obtain: each of $A_{0}=R_{1} \cup$ $F_{1} \cup\left[P \cap\left(R_{0} \cup F_{0}\right)\right], \quad A_{1}=S_{2} \cup F_{2} \cup\left[P \cap\left(S_{0} \cup F_{0}\right)\right], \quad A_{2}=S_{2} \cup F_{2} \cup[Q \cap$ $\left.\left(S_{0} \cup F_{0}\right)\right]$ and $A_{3}=R_{1} \cup F_{1} \cup\left[Q \cap\left(R_{0} \cup F_{0}\right)\right]$ are simple continua in $X$. Then $A_{0} \cap A_{1}=P \cap F_{0}, A_{2} \cap A_{3}=Q \cap F_{0}, A_{0} \cap A_{3}=R_{1} \cup F_{1}$ and $A_{1} \cap A_{2}=S_{2} \cap F_{2}$ and thus no three of the $A_{i}$ 's have a point in common. This completes the proof of (i) implies (ii).

Now (i) implies (iii) follows from the above if we let $B_{1}=A_{0} \cap A_{3}$ and $B_{2}=A_{1}$ $\cap A_{2}$ since $B_{1}=R_{1} \cap F_{1}$ and $B_{2}=S_{2} \cup F_{2}$ are simple continua in $X$ and $B_{1} \cup B_{2}$ separates $X$. Furthermore, (iii) implies (ii), by the proof of (i) implies (ii), for in it we merely assumed there existed disjoint simple continua, say $B_{1}$ and $B_{2}$ (or $C$ and $D$ ), whose union separates $X$.

Hence, it remains to show that (ii) implies (i). But this is easy, for if $L=A_{0} \cup A_{1}$ and $M=A_{2} \cup A_{3}, L$ and $M$ are continua and $L \cap M$ is a separated closed set. Thus $X$ is multicoherent and this completes the proof of the corollary.

Remark (3.3). Note that Corollary (3.2) has Theorem (3.1) as an easy consequence: For suppose every pair of disjoint continua in $X$ can be separated by a continuum and $X$ is not unicoherent. Then by Corollary (3.2), $X=A_{0} \cup A_{1} \cup A_{2} \cup$ $A_{3}$ as in (3.2)(ii). But $A_{0}$ and $A_{3}$ cannot be separated by a continuum, so $X$ must be unicoherent.

Remark (3.4). The circularity, $m(X)$, of $X$ is defined by: $m(X)=\sup \{n \in \mathbf{N}$ : $X=\cup\left\{A_{i}: 0 \leqslant i<n-1\right.$ where each $A_{i}$ is a nonempty continuum, no three $A_{i}$ 's have a point in common, and $A_{i} \cap A_{j} \neq \varnothing$ if and only if $\left.\left.|(i-j) \bmod n| \leqslant 1\right\}\right\}$. A. H. Stone showed that $m(X) \geqslant 3$ if $X$ is multicoherent [10]; it is known that $m(X) \geqslant 6$ if $X$ is multicoherent and compact, and $m(X)=\infty$ if $X$ is weakly-finitely multicoherent [5]. Furthermore, if $X$ is a planar, Peano continuum, then $m(X)=\infty$ or $m(X)$ is an even integer [1]. There exists a planar Peano continuum for which $m(X)=6$ [2]; in [3], an example $D$ where $m(D)=4$ is given.

Corollary (3.2) and Theorem (3.1) are each equivalent to the following: $X$ is multicoherent if and only if $m(X) \geqslant 4$; the construction of $\left\{A_{0}, A_{1}, A_{2}, A_{3}\right\}$ in Corollary (3.2) shows that for a multicoherent space $X, m(X) \geqslant 4$. (We acknowledge that it is redundant to speak of two theorems as equivalent, however, before the present paper, only special cases (e.g. $X$ is compact [5], or $X$ is perfectly normal [4]) were known.)

The example $D$ of [3] (where $m(D)=4$ ) shows that Corollary (3.2) (and its equivalent statements) is a best possible result in the following sense: There exists a multicoherent space $D$ such that whenever $B_{1}, B_{2}$ and $B_{3}$ are pairwise disjoint simple continua, one of the pairs, $B_{1} \cup B_{2}, B_{2} \cup B_{3}$ or $B_{3} \cup B_{1}$, fails to separate $X$. (For if there existed a triple $B_{1}, B_{2}$ and $B_{3}$ so that all the pairs $B_{1} \cup B_{2}, B_{2} \cup B_{3}$ and $B_{3} \cup B_{1}$ separated $X$, then $m(D) \geqslant 6$ [5, Theorem 3]; of course this is not true in general, since $m(D)=4$.)

\section{REFERENCES}

1. Harold Bell, Ezra Brown, R. F. Dickman, Jr. and E. L. Green, Circularity of graphs and continua: Topologv, Fund. Math. 112 (1981), 103-110.

2. Harold Bell and R. F. Dickman, Jr., A counterexample to a conjecture of A. H. Stone, Proc. Amer. Math. Soc. 71 (1978), 305-308. 
3. The end of a conjecture of A. H. Stone. Topology Appl. (submitted).

4. R. F. Dickman, Jr., A proof of a conjecture of A. H. Stone, Proc. Amer. Math. Soc. 80 (1980), 177-180.

5. Multicoherent spaces, Fund. Math. 91 (1976), 219-229.

6. R. F. Dickman, Jr. and L. R. Rubin, C-separated sets and unicoherence, Fund. Math. 83 (1973), $25-33$.

7. J. H. V. Hunt, The Phragmen-Brouwer theorem for separated sets, Bol. Soc. Sci. Mexicana 19 (1974), 26-35.

8. J. H. V. Hunt and E. D. Tymchatyn, $A$ theorem on involutions on unicoherent spaces, Quart. J. Math. Oxford Ser. (2) 32 (1981), 57-67.

9. Leonard Gillman and Meyer Jerison, Rings of continuous functions, Van Nostrand Rheinhold, Princeton, N.J., 1960.

10. A. H. Stone, Incidence relations in unicoherent spaces, Trans. Amer. Math. Soc. 65 (1949), 427-447.

11. ___ Incidence relations in multicoherent spaces. I, Trans. Amer. Math. Soc. 66 (1949), 389-406.

12. __ Incidence relations in multicoherent spaces. II, Canad. J. Math. 2 (1950), 461-480.

13. Incidence relations in multicoherent spaces. III, Pacific J. Math. 2 (1952), 99-126.

14. G. T. Whyburn, Analvtic topologv, Amer. Math. Soc. Colloq. Publ., vol. 28, Amer. Math. Soc., Providence, R.I.. 1942.

Department of Mathematics, Virginia Polytechnic Institute and State University, BlacksBURG, VIRGINIA 24061 